\title{
AS PRATICAS DE EDUCAÇÃO EM SAÚDE COM ENFOQUE DA DIABETES NA CAPACITAÇÃO DOS AGENTES COMUNITÁRIOS - ACS, EM UM PROJETO DE EXTENSÃO: Relato de experiência
}

\author{
Vinicius Lino de SOUZA NETO ${ }^{1}$ \\ Nathália Araújo da NÓBREGA ${ }^{2}$ \\ Gabrielly de Carly Pereira de CARVALHO ${ }^{3}$ \\ Renata Laís da Silva NASCIMENTO ${ }^{4}$ \\ Géssica Raíssa Francisco FRANÇA ${ }^{5}$ \\ José JUSTINO FILHO**
}

\begin{abstract}
${ }^{1}$ Discente do Curso Bacharelado em Enfermagem da Universidade Federal de Campina Grande e Bolsita do Programa de Extensão - PROBEX / Vigência - 2013 - UFCG; Líder do Grupo de pesquisa em Epidemiologia e Saúde Coletiva - GPESC. E-mail: vinolino@ hotmail.com

${ }^{2}$ Discente do Curso Bacharelado em Enfermagem da Universidade Federal de Campina Grande e Membro do Grupo de pesquisa em epidemiologia e saúde coletiva - GPESC - UFCG; Colaborada do projeto de Extensão PROBEX/ Vigência - 2013. E-mail: nathaliaanobrega@ hotmail.com
\end{abstract}

${ }^{3}$ Discente do Curso Bacharelado em Enfermagem da Universidade Federal de Campina Grande e Membro do Grupo de pesquisa em epidemiologia e saúde coletiva - GPESC - UFCG; Colaborada do projeto de Extensão PROBEX/Vigência - 2013. E-mail: gabriellycarly@gmail.com

${ }^{4}$ Discente do Curso Bacharelado em Enfermagem da Universidade Federal de Campina Grande e Membro do Grupo de pesquisa em epidemiologia e saúde coletiva - GPESC - UFCG; Colaborada do projeto de Extensão PROBEX/ Vigência - 2013. E-mail: renata-blink@ @otmail.com

${ }^{5}$ Discente do Curso Bacharelado em Enfermagem da Universidade Federal de Campina Grande e Membro do Grupo de pesquisa em epidemiologia e saúde coletiva - GPESC - UFCG; Colaborada do projeto de Extensão PROBEX/ Vigência - 2013. E-mail: g.raissa.fran@ gmail.com

**Coordenador do Projeto de Extensão - PROBEX/ Vigência- 2013, intitulado "Práticas de Educação em Saúde frente aos portadores de diabetes mellitus assistidos nas UB'S, zona urbana de Cuité: Uma promoção da Saúde.”. Mestrado e Doutorado em Sociologia pela Universidade Federal da Paraíba. Professor Adjunto IV do Curso de Enfermagem da Universidade Federal de Campina Grande - UFCG/ Campus- Cuité, Paraíba (PB), Brasil. E-mail: josejustino@bol.com.br

**Endereço para correspondência do autor:

Sitio olho D'água da bica, SIN, CEP: 58.175-000 Tel: 3372-1900. Professor do Curso de Graduação em Enfermagem da Universidade Federal de Campina Grande - UFCG/ Campus - Cuité.

\section{Recebido em: 11/09/2013 - Aprovado em: 13/12/2013 - Disponibilizado em: 15/01/2014}

RESUMO - Objetivo: Relatar a vivência das praticas de educação em saúde dos estudantes de enfermagem em um projeto de extensão. Metodologia: Trata-se de um estudo descritivo do tipo relato de experiência, das vivências dos discentes de Enfermagem no processo de articulação das praticas de educação em saúde aos agentes comunitários das unidades básicas. Resultados: Neste ensejo, a experiência se deu com os agentes comunitários das 05 unidades básicas de saúde, zona urbana, do município de Cuité, $\mathrm{PB}$, ao qual foi vivenciada através de uma capacitação sobre a diabetes, sendo esta transcorrida em momentos, onde no primeiro momento, foi explanado o significado da diabetes, as suas etiologias, fatores de risco, tipos, comorbidades secundarias, como a nefropatia, retinopatia e neuropatia diabética, logo em seguida uma dinâmica sobre a temática e sucessivamente em outro momento foram feitas perguntas sobre tudo que tinha sido discutido. Conclusão: Percebe-se que a prática de educação em saúde pode ser articulada de varias formas, como oficina, gincana, palestras, capacitação entre outros, onde o desenrolar destas ações se transcorrer de maneira dinâmica, sem uma metodologia de depósito as informações, proporciona um maior entendimento ao publico alvo.

Palavras-chave: Educação em Saúde. Atenção Primaria à saúde. Enfermagem. Comunidade. Agente Comunitário de Saúde. 


\title{
THE PRACTICE OF HEALTH EDUCATION OF DIABETES WITH FOCUS ON TRAINING OF STAFF COMMUNITY - ACS, A PROJECT IN EXTENSION: experience report
}

\begin{abstract}
Objective: To report the experience of the practice of health education of nursing students in an extension project. Methodology: This is a descriptive study reporting experience, the experiences of nursing students in the process of articulation of the practice of health education on the Community of the basic units. Results: In this opportunity, the experience took place with community agents of 05 basic health units, the urban area of the municipality of Cuité, PB, which was experienced by a training on diabetes, which is spent with moments where the initially, it was explained the meaning of diabetes, its causes, risk factors, types, secondary comorbidities, such as nephropathy, retinopathy and diabetic neuropathy shortly thereafter on a dynamic theme and then in another moment were asked questions about everything which had been discussed. Conclusion: It is clear that the practice of health education can be articulated in many ways, as a workshop, gymkhana, lectures, training among others, where the conduct of these actions are dynamically elapse without a method of filing information, provides a greater understanding of the target audience.
\end{abstract}

Keywords: Health Education. Primary health care. Nursing. Community. Agent Community Health.

INTRODUÇÃO

O Diabetes Mellitus (DM) é uma doença crônica não transmissível de grande relevância para a saúde pública e para a sociedade, independentemente do grau de desenvolvimento econômico do pais. No Brasil, segundo estudo Multicêntrico Brasileiro, Romero, Barceló e Machado (2001) 12,4 milhões de pessoas foram acometidos por DM no ano de 2011, e a previsão é que este número aumente para 19,6 milhões de pessoas até 2030. Dentre os tipos de DM, o tipo 2, compreende $90 \%$ dos agravos presentes no mundo e está intimamente relacionado com o excesso de peso e o sedentarismo, conforme retrata o estudo multicêntrico supracitado.
$\mathrm{O}$ aumento da prevalência do diabetes, aliado à complexidade de seu tratamento, tais como restrições dietéticas, uso de medicamentos e complicações crônicas associadas (retinopatia, nefropatia, neuropatia, cardiopatia, pé neuropático, entre outras) reforçam a necessidade de programas educativos eficazes e viáveis aos serviços públicos de saúde. A mudança de comportamentos, com a adoção de uma dieta balanceada e da prática de atividades físicas, é essencial para que o controle e o tratamento do DM tenham êxito (SILVA et al., 2009). Para Rocha, Moreira e Rodrigues (2005) mostra que o controle e a prevenção de complicações do diabetes são possíveis por meio de programas educativos. 
Nesta perspectiva as ações educativas para o autocuidado em DM, quando conduzidas de forma qualificada, com suas competências delineadas no processo de aprendizagem contribuem para o melhor controle metabólico do indivíduo, pois cabem tantos aos profissionais de saúde, principalmente da atenção básica, como o cliente a responsabilidade de produzir as condições favoráveis ao processo de aquisição de conhecimentos sobre o DM, que possam levar à mudança nos hábitos de vida e manejo da doença, para que assim tenha uma qualidade de vida sinérgica (FAEDA; PONCE DE LEON, 2006).

\footnotetext{
A utilização de práticas educativas como estratégia no tratamento do DM tem como objetivo melhorar o conhecimento do indivíduo sobre o DM e seu acompanhamento, assim como levar a hábitos de vida saudáveis, que melhorem a qualidade de vida, aumentando a sua autonomia perante a doença. Uma característica dessas práticas é a possibilidade de unir pessoas com histórias parecidas, que compartilharão experiências, com a possibilidade de aprimorar o conhecimento, modificar as atitudes e habilidades que favorecerão a mudança de comportamento para a melhora do
}

controle da doença e qualidade de vida (BIRAL; CARDOSO; GRUNSPAN, 2005).

Diante do exposto, a proposta em questão tem como objetivo explanar a vivência dos extensionista do projeto, intitulado "Práticas de Educação em Saúde frente aos portadores de diabetes mellitus assistidos nas UB'S, zona urbana de Cuité: Uma promoção da Saúde", na articulação das práticas de educação em saúde no processo de capacitação aos agentes comunitários da atenção primária, com enfoque na referida temática.

\section{METODOLOGIA}

O estudo em questão é de teor qualitativo e descritivo, tipo de relato de experiência, para Minayo (2007) a abordagem vivencial, ou seja, o relato de experiência permite descrever situações ao quais os autores da pesquisa fez parte. Já Cervo e Bervian (2007) denota que o estudo descritivo é aquele em que se observa, registra, analisa e correlacionam fatos e fenômenos variáveis sem manipulá-los. Lima- Costa e Barreto (2003) discute que os estudos descritivos têm por objetivo determinar a distribuição de doenças ou condições relacionadas à saúde segundo o tempo, o lugar e as 
características dos indivíduos. Ou seja, responder às perguntas quando, onde $\mathrm{e}$ com quem acontece o fenômeno a ser estudado.

As experiências relatadas foram vivenciadas pelos discentes do curso bacharelado em enfermagem da Universidade Federal de Campina Grande - UFCG/ campus Cuité, no período de Julho á Agosto de 2013, ao qual fazem parte do projeto de extensão, onde o projeto seguiu todos os trâmites legais, conforme edital $\mathrm{N}^{\circ} 01 / 13$, sendo aprovado pela Pro reitoria de Ensino e Extensão da presente instituição.

Neste segmento, o cenário das ações do projeto foi as 05 Unidades Básicas de Saúde da zona urbana do município de Cuité, ao qual para cada, existe uma equipe de saúde da família. Em suma, na região urbana tem o quantitativo de 41 agentes comunitário de saúde. Desta forma, conforme dados do programa Hiperdia, o município possui 312 diabéticos, com faixa etária de 35 á 75 anos, desmembrados entre as 05 unidades (BRASIL, 2013).

O principal objetivo do projeto é contribuir para educação em saúde diante dos portadores de diabetes, no que se refere às mudanças sociais $\mathrm{e}$ fisiológicas que acometem o individuo e como adaptalas ao seu cotidiano, mas para chegar ao feito, ou seja, está próximo ao nosso publico alvo, é necessário acrescentar um interlocutor entre as partes, extensão e comunidade, ao qual o ACS é o grande articulador/interlocutor deste ato. Com isso, a ação promoveu um aperfeiçoamento aos conceitos sobre a diabetes para que os agentes tornem-se vigilantes e promulgadores de saúde, aplicando a promoção, prevenção e proteção ao diabético.

\section{RELATO DE EXPERIÊNCIA}

A prática da extensão leva a um caminho entre o acadêmico e a população, permeando uma troca de saberes e vivências, que jamais serão abstraídas de cada um. Conforme supradescrito anteriormente, o óbice do projeto é articular praticas de educação em saúde voltado ao portador da diabetes no referido município, pois o lócus de atuação segundo Brasil (2013), está entre os piores índices de desenvolvimento das atividades do Sistema Único de Saúde (IDSUS-2009), quando se comparado aos municípios que compõem o Curimatau - Paraibano/ PB.

Neste sentido, para dedilharmos o caminho ao portador da diabetes, precisaria conhecer o elo entre o serviço 
da atenção primária e o mesmo, ou seja, o agente comunitário de saúde, com isso primou-se em aperfeiçoar os conhecimentos destes através de uma capacitação, para que assim fosse renovado os seus conhecimentos frente à temática e aperfeiçoando a sua abordagem ao portador (a). Ressaltando que os meios de articulação da capacitação, ou seja, as praticas de educação em saúde foram às mesmas para a 05 unidades básicas. Desta forma, a ação transcorreu de forma dinâmica em três momentos, conforme descrito abaixo:

No primeiro momento, foi explanado por meio de slides, em uma linguagem coloquial pouco cientifica, mas não se abstendo da mesma, sobre o significado da diabetes, as suas etiologias, fatores de risco, tipos, comorbidades secundarias, como a nefropatia, retinopatia e neuropatia diabética e pôr fim discorreu sobre a diabetes nos segmentos sociais, criança, adolescente, idoso e gestante. Dentro das varias causas secundárias conforme supradescrito se deu ênfase ao pédiabético, decorrente da alta incidência do mesmo em todas as unidades, ao qual a faixa etária dos portadores de diabetes ficou entre 65 á 75 anos, ou seja, uma população idosa, onde impera o processo de senilidade e senescência do envelhecimento.

No discorrer da ação, já em um segundo momento foi levado pelos extensionistas os alimentos de maior facilidade de acesso e conhecimento que os agentes e a comunidade detém, como por exemplo, o refrigerante (cocacola lata $-350 \mathrm{ml}$ ), biscoito recheado, gelatina, suco e bebida láctea industrializado, entre outros. Logo após a apresentação dos alimentos, foi questionado aos mesmos se sabiam o quantitativo de colheres de sopa de açúcar equivalente para cada alimento, muitos optaram, quando revelado o quantitativo ficaram surpresos com o teor de açúcar. Desta forma, a dinâmica teve como objetivo alerta- lós quanto a pratica da má alimentação e como ela é prejudicial ao nosso corpo, dando segmento as ações foi passado um vídeo que explanava por meio de figuras ilustrativas o acometimento da diabetes.

Para o fim das ações, em um terceiro momento, foi construída a caixinha das perguntas pelos colaboradores do projeto, ao qual cada agente retirava um papel e nele tinha uma pergunta sobre toda a temática discutida no processo de capacitação, as resposta foram surpreendentes, pois o óbice era analisar se o feito teve uma 
absorção positiva. Em seguida, abrimos um momento para dúvidas, críticas e sugestões, alguns questionamentos quanto à diabetes foram esclarecidos, onde os agentes relataram que a dinamicidade do evento, tirou a imagem das ações enfadonhas e de sobrecarga de informação. Ressaltando que, para todas estas articulações foi solicitado o aval a gestão de atenção básica do município, ao qual membros da mesma se fez presente em todas as capacitações.

\section{DISCUSSÃO}

Neste ensejo, as ações de articulação das práticas de educação em saúde desenvolvida pelos extensionistas, proporcionou uma vivência singular para o grupo, pois percebemos as particularidades do saber de cada agente de saúde e como os mesmos tinham o seu olhar sobre a temática, ao qual foi lapidado em cada momento, por isso é substancial esse feedback que a extensão pode proporcionar entre as comunidades cientificas e popular.

Desta forma, a DM é uma doença que necessita de mudanças que duram toda a vida e seu tratamento exige uma terapia médico nutricional, medicações diárias, exercícios físicos programados, automonitorização da glicemia, cuidados com o corpo, educação permanente e modificação comportamental. Desse modo, torna-se necessário uma ação educativa para instruir e conscientizar o diabético da importância do seu conhecimento sobre a DM como parte integral do cuidado, proporcionando um melhor convívio com a doença, tornando-o protagonista de seu tratamento e, assim controlando a patologia e suas complicações, ao qual o agente comunitário de saúde é um articulador destas ações de prevenção e promoção da saúde no âmbito da atenção primária. (BIRAL; CARDOSO; GRUSPAN, 2005).

Neste aspecto, acredita-se que as ações educativas, junto ao paciente, família e comunidade, têm um papel essencial no controle dessa enfermidade, uma vez que suas complicações estão estritamente ligadas ao conhecimento para o cuidado pessoal diário adequado e ao estilo de vida saudável. O diabético, em especial, necessita ser estimulado pelos profissionais de saúde a manter uma vida independente, adaptando- se da melhor maneira possível às modificações exigidas para o controle metabólico. Os profissionais de saúde têm a responsabilidade de auxiliar os 
portadores de DM ensinando-lhes o autocuidado, instruindo-os sobre a doença e conscientizando-os da importância da automonitorização contínua da glicemia, de modo a garantir mudança de comportamento e participação no tratamento (TORRES, HORTALE, SHALL, 2005).

As ações educativas contribui para uma melhor qualidade de vida; entretanto, algumas questões devem ser consideradas para se obter resultados efetivos, como a fase do ciclo vital e suas peculiaridades. Tendo em vista que a conscientização e a educação são fatores fundamentais para um adequado controle e prevenção da doença. Atualmente, vivencia-se um aumento tanto na expectativa de vida, quanto na maior incidência das doenças crônicas não transmissíveis. Considera-se, pois, fundamental que os profissionais de saúde, direcionem sua prática profissional para ações que levem à independência, à autonomia e à qualidade de vida aos diabéticos. Para tanto, é necessário ampliar, cada vez mais, o conhecimento sobre, as doenças crônicas e os fatores que têm determinados melhores ou piores condições de saúde e qualidade de vida (FAEDA; PONCE DE LEON, 2006)
Os profissionais de saúde de diversos segmentos da interdisciplinaridade assistencial devem estar atentos às mudanças que estão ocorrendo no país e no mundo, para que possa adequar seu conhecimento teórico-prático às reais necessidades de saúde da população proporcionando uma assistência equalizada e holística diante do portador de diabetes, tendo como mecanismo trópico a educação em saúde (KNOWLER et al., 2002).

O desenvolvimento das práticas de educação em saúde juntamente com o trabalho em equipe é visto como uma habilidade capaz de consolidar as ações almejadas e a busca aos objetivos. Neste contexto, o processo de educação em saúde, torna-se uma necessidade contemporânea e iminente ao contingente populacional, pois através deste veículo reintegramos o ser tangenciado socialmente e introjetamos o mesmo no epicentro do processo de globalização, ou seja, na assistencialização das políticas públicas.

\section{CONSIDERAÇÕES FINAIS}

Contudo, após a explanação da vivência dos extensionistas, percebe-se que a prática de educação em saúde pode ser articulada de várias formas, como oficina, gincana, palestras, 
capacitação. É notório que na experiência acima descrita frente aos agentes comunitários de saúde leva-os a serem multiplicadores de ações no que tange a promoção, prevenção e proteção à saúde principalmente aos pacientes portadores de doença crônica não transmissível, como a diabetes, ao qual a sua incidência vem tomando proporções significativas no âmbito de acometimento.

Os ACS atuam incessantemente na vigilância a saúde do contingente populacional, pois para uma articulação de ações de educação em saúde com qualidade é fundamental o desenvolvimento da educação continuada, ao qual proporciona aos mesmos um aprimoramento do

\section{REFERÊNCIAS}

BIRAL, A. M.; CARDOSO, P. M.; GRUNSPAN, S. A importância do educador em diabetes mellitus.

Diabetes Clínica, São Paulo, v. 9, n. 3, p.193-99, 2005. Disponível: Acesso em: 10 de Jul.2013.

BRASIL. Ministério da Saúde. Sistema de Informação da Atenção BásicaSituação de Saúde-Paraíba. Disponível: http://tabnet.datasus.gov.br/cgi/tabcgi.e xe?siab/cnv/SIABSPB.def Acesso em: 07 de Jul.2013. conhecimento tanto ao portador de diabetes como as demais doenças crônicas transmissíveis, ou não, levando também ao saber das novas tecnologias assistenciais a essas enfermidades. Por isso, que a fusão simbiótica entre extensão cientifica e comunidades levam não só aprimorar o saber que se tem, mas conhecer e questionar o que desconhece.

Neste contexto, nos deparamos frente à necessidade da criação de programas de capacitação tanto em esfera municipal, estadual e federal, para os profissionais da área de saúde, pois a cada dia que se passa novos mecanismos assistências são adicionados e/ou substituindo os antigos modelos.

CERVO, A. L.; BERVIAN, P. A.

Metodologia Científica. 4. ed. São

Paulo: Ed. Prentice Hall, 2007.

FAEDA, A.; PONCE DE LEON, C. G. R. M. Assistência de enfermagem a um paciente portador de Diabetes Mellitus. Rev. Bras. Enferm, v. 59, n.6, p. 81821, 2006. Disponível: http://www.scielo.br/scielo.php?pid=S0 034-

71672006000600019\&script=sci_arttex t Acesso em: 07 de Jul.2013

LIMA-COSTA, M.F.; BARRETO, S. M. Tipos de estudos epidemiológicos: conceitos básicos e aplicações na área do envelhecimento. Epidemiol. Serv. 
Saúde, Brasília, v. 12, n. 4, p. 189201,2003. Disponível: http://scielo.iec.pa.gov.br/pdf/ess/v12n4 /v12n4a03.pdf Acesso em: 14 de Jul.2013.

MINAYO, C. de S. (org.). Pesquisa social: teoria, método e criatividade. $26^{\mathrm{a}}$ ed. Petrópolis: Vozes, 2007.

ROCHA, J. M.S; MOREIRA, T. M. M; RODRIGUES, D. P. Adaptação do cliente com Diabetes Mellius tipo 2 à doença e o tratamento. Rev. Rene. Fortaleza, v.6, n.1, p.20-28, 2005. Disponível:

http://www.revistarene.ufc.br/revista/in dex.php/revista/article/view/778/pdf. Acesso em: 07 de Jul.2013.

ROMERO, B. B.; BARCELÓ, A.; MACHADO, C. A. Campanha nacional de detecção de casos suspeitos de diabetes mellitus no Brasil: relatório preliminar. Rev Panam Salud Publica, v.10, n.5, p.318-27, 2001. Disponível em:

http://www.scielosp.org/pdf/rpsp/v10n5 17353.pdf Acesso em: 18 de Jul.2013.

SILVA, A. R. v et al. Educação em saúde a portadores de diabetes mellitus tipo 2: revisão bibliográfica.Rev. Rene. Fortaleza, v. 10, n. 3, p. 146-151,2009. Disponível:

http://www.revistarene.ufc.br/revista/in dex.php/revista/article/view/554/pdf.Ac esso em: 18 de Jul. 2013.

TORRES, H.C; HORTALE, V.A;

SHALL, V.T. Validação dos questionários de conhecimento (DKNA) e atitude (ATT-19) de Diabetes Mellitus. Rev Saúde Publica, v. 39, n.6, p. 906-11, 2005. Disponível: http://www.scielo.br/pdf/rsp/v39n6/269 84.pdf. Acesso: 20 de Jul.2013.

KNOWLER, W.C et al. Diabetes Prevention Program Research Group. Reduction in the incidence of type 2 diabetes with lifestyle intervention or metformin. N Engl J Med, v.346, n.6, p.393-403, 2002. Disponível:

http://www.nejm.org/doi/full/10.1056/N

EJMoa012512 Acesso em: 30 de

Jul.2013. 